\title{
ERRATUM
}

\section{Erratum zu: Psychopathie, antisoziale Persönlichkeitsstörung und Sexualdelinquenz}

\author{
Safiye Tozdan ${ }^{1} \cdot$ Peer Briken ${ }^{1}$ \\ Online publiziert: 25 . Juli 2018 \\ ๑) Springer-Verlag GmbH Deutschland, ein Teil von Springer Nature 2018
}

\section{Erratum to: Psychopathy, antisocial personality disorder and sexual delinquency}

\section{Erratum zu:}

Forens Psychiatr Psychol Kriminol 2018

https://doi.org/10.1007/s11757-018-0482-3

Die Originalversion dieses Beitrags enthielt einen Fehler: Die Reihenfolge der Autorennamen war falsch wiedergegeben. Die richtige Reihenfolge ist oben dargestellt.

Die Online-Version des Originalartikels ist unter https://doi.org/ 10.1007/s11757-018-0482-3 zu finden.

$\triangle$ Prof. Dr. med. Peer Briken briken@uke.de

1 Institut für Sexualforschung und Forensische Psychiatrie, Universitätsklinikum Hamburg-Eppendorf, Martinistr. 52, 20246 Hamburg, Deutschland 